\title{
Changes in the perception of spatial location: A test of potentiation vs. recalibration theory
}

\author{
RICHMOND WILLEY, JOHN W, GYR, and ADELE HENRY \\ University of Michigan, Ann Arbor, Michigan 48109
}

\begin{abstract}
Two experiments tested recalibration and muscle potentiation theories of adaptation to prismatic displacement of the visual field. Each experiment included a condition in which only recalibration could occur and another condition in which only potentiation was possible. In one experiment, the displacement was simulated on a computer-driven cathode ray tube, and in the other, the displacement was produced by actual prisms. In both experiments, significant adaptation occurred only in the potentiation condition. Implications of this finding for recent criticisms of potentiation theory are discussed.
\end{abstract}

This study presents data which are relevant to the question raised by Ebenholtz (1974) and Paap and Ebenholtz (1976) as to whether changes in the visual perception of location, which are induced by visual rearrangement, are to be interpreted by the hypothesis of central visual learning (e.g., Epstein, 1975; Rock, 1966; Wallach \& Frey, 1972) or by the hypothesis of muscle potentiation (e.g., Ebenholtz, 1974; Paap \& Ebenholtz, 1976).

The visual learning hypothesis has been used to explain the results of studies in which the perceived location of visual targets has been rearranged through the use of prisms. For example, if an observer wears a base-left prism while walking through a hallway, all visual targets are perceptually displaced to the right. Consequently, if observers are to focus on actually straight-ahead targets, and thus visually guide their locomotion down the center of the hall, they have to turn their eyes and/or their heads to the right. Subjects who experience this kind of training demonstrate adaptation, in that a comparison of preand posttest settings of a target to look straight ahead reveals a shift in the direction of prism displacement.

According to the visual learning theorists, the factors which cause this adaptation are the following. Normally there exist several cues for the spatial localization of a target. Some of these are visual, others may be of a proprioceptive and/or efferent character. When a discrepancy exists between such classes of cues-as when the visual cues are systematically rearranged by means of a prism-one or more classes of cues may become recalibrated. Thus, in the above example of training with a base-left prism, there might be visual-motor recalibration, which means that the eye, when it now seems to the subject to be aiming straight ahead, is actually looking to the right of straight ahead.

By contrast, the theory advanced by Ebenholtz (1974) and Paap and Ebenholtz (1976) suggests that this same adaptation can be explained by the peripheral effects of muscle potentiation, which is a hypothesized involuntary and unconscious component of innervation to the eye muscles induced by sustained off-center eye position. Consider, again, the case of an observer walking down a hallway while looking through a base-left prism. As this will displace objects to the right and induce a tendency in subjects to keep their eyes directed to the right for the duration of the exposure, potentiation favoring rightward orientation of the eyes will be generated. To the extent that visual straight ahead is determined by registered eye position, this training will result in posttest settings of straight ahead which are displaced to the right relative to the pretest, since subjects will be looking further to the right than they think they are. Muscle potentiation theory thus predicts effects in the same direction as does perceptual learning theory, and accordingly, these effects are confounded by typical adaptation experiments.

Paap and Ebenholtz (1976) have begun experiments designed to answer the question of which of the hypothesized processes is responsible for the obtained adaptation or whether both theories are valid. They have gone about this by predicting, on the basis of potentiation and on the basis of recalibration theory, which factors would most strongly advance the decay in the adaptation, and then set out to test these predictions.

The experiment they conducted involved visual rearrangement. They showed that training, subsequent to exposure, which required subjects to alternate left and right eye movements in rapid succession, was a more potent means for reducing adaptation than, say, returning subjects to a visually normal environment. This finding would appear to strongly favor potentiation theory.

A more direct experiment to test the validity of the potentiation theory vs. the recalibration theory 
would involve studying not the decay in adaptationas Paap and Ebenholtz have done-but the adaptation itself. If the discrepancy between various cues for spatial location, which is the cornerstone of the perceptual learning argument, is to be disentangled from the process of potentiation, the following two experimental conditions may be contrasted. In Condition 1, visual rearrangement is produced such that subjects learn to point off center at a target that looks straight ahead. This condition thus provides a chance for perceptual learning, since there is cue discrepancy, but it eliminates the chance for muscle potentiation to manifest itself, inasmuch as subjects look straight ahead during exposure. Condition 2 , on the other hand, is designed such that there is no visual rearrangement, and hence no perceptual change due to perceptual learning. However, this condition requires subjects to point at, and focus on, a target that is off center, thus inducing muscle potentiation. Two experiments involving these two conditions will now be reported.

\section{EXPERIMENT 1}

\section{Method}

Subjects. The subjects were male and female college students drawn from a subject pool. All were required to have normal vision, with or without glasses. Fifteen subjects were used in each condition. Three subjects were rejected, one for violating instructions and two because of extremely unstable judgments resulting from autokinetic movement of the stimulus.

Apparatus. The main portion of the apparatus consisted of a cathode ray tube display (CRT) operated by a PDP-9 computer. The CRT was located inside a completely dark room. On a smal table in front of the CRT, $47 \mathrm{~cm}$ from the CRT screen, a biteboard was mounted, and also a button box with which the subject could vary the horizontal location of a stimulus on the screen so as to indicate when it looked straight ahead. There was also a light pen, the horizontal location of which would be registered when the subject pointed with it at the screen. A hood surrounded the biteboard; this hood contained an aperture, which restricted the subject's view to a small portion of the screen, and a filter, which prevented the afterglow on the screen from being seen. Extending from the hood to the center of the screen was a horizontal panel; the subject could reach up under this to the screen without seeing his hands. Stimuli were small squares and crosses, about $2 \mathrm{~mm}$ in height and width, located just above the barrier. With the lights out, the subject could see the stimuli clearly, but could see no clear boundaries in the visual field, even after dark adaptation.

No actual prism was used in this experiment. The computer simulated prism displacement by taking the location of the light pen hit (below the barrier) and adding a constant to it to determine the horizontal location of the feedback (seen above the barrier). This was one reason for a totally dark visual field; this simulation could only work for stimuli generated on the screen. The other reason for such a field was that the perception of a point as straight ahead might have been influenced by visual boundaries.

Procedure. Both conditions involved a pretest, a training period, and a posttest identical to the pretest. In the pre- and posttests, the subjects, using the button box, set a small square to look straight ahead. In training, the subjects held the light pen with both hands and pointed at a small cross, receiving a small square as feedback. The subjects read a written description of the experiment while the biteboard was being made. The pre-posttest, and the training procedure were described as Task A and Task B, respectively, and it was emphasized that in Task $A$ the subject should not reason or calculate in any way, or use a mechanical rule for the adjustments.

After reading the instructions, the subjects were taken to the experimental room and allowed to familiarize themselves with the apparatus by means of a few practice trials. The experiment began with 10 pretest trials, which (unknown to the subject) actually constituted further practice, and also an opportunity for dark adaptation. After a brief rest, the experiment proper began with 24 pretest trials. In both pre- and posttests, the starting positions of the stimuli alternated from left to right and varied in absolute position over a small range (about $1 \mathrm{~cm}$ ). There was a longer rest period after the pretest.

Training consisted of 80 trials in both conditions. Target stimuli varied over a moderate range (about $2 \mathrm{~cm}$ ), and the sequence of stimulus locations relative to each other was the same in both conditions. However, the absolute locations of stimuli were different in the two conditions. In Condition 1, the center of the stimulus range was equal to the mean of the subject's pretest; in Condition 2, the center of the range was displaced to the right of the pretest mean by an amount equal to the simulated prism displacement in Condition 1. This displacement was $6.69^{\circ}$ with feedback displaced to the right of the light pen tip. Thus, in Condition 1, the subject was looking at targets which should have appeared more or less straight ahead to him, while having to learn to point his hands to the left in order to get the feedback on target, while in Condition 2 the subject saw targets displaced to the right while having to learn to point his hands to the right. Note that, while the instructions to the subjects were the same in both conditions (try to get the feedback on target), the function of the task was different, depending on which theoretical expectation was being tested. In Condition 1 , the task was meant to induce a discrepancy between felt arm position and perceived visual location, while in Condition 2, the task merely induced the subject to look to one side, with the arm movement serving no function (according to potentiation theory) other than maintaining subjects' interest.

After training, the subject was briefly reminded to do the next task exactly as he had done the pretest; i.e., to make settings that looked correct, without reasoning or calculating. The posttest began immediately and consisted of 24 trials with the same sequence of starting positions as the pretest. After the experiment, the subject was asked whether he had, in fact, done the pretest and posttest in the same way, and in particular whether he had derived any information from training which consciously influenced his posttest judgments (such as concluding, in Condition 2, that the location of the training targets represented the true straight-ahead position). Subjects who reported a conscious influence were rejected.

\section{Results}

The results are reported in CRT raster units (the minimum distance between points on the screen). For the observation distance of $47 \mathrm{~cm}, 35$ raster units equal about $1^{\circ}$. For each subject, the pretest mean was subtracted from that of the posttest to create a single score. Both theories predict a positive value for this score, which represents posttest settings to the right of those in the pretest. The individual scores, with the group means and standard deviations, are shown in Table 1. A $t$ test of the difference between the groups was performed, and also $t$ tests of the difference of each group mean from zero. For the difference test, $t(28)=2.99, p<.01$. The 
Table 1

Experiment 1 Adaptation Scores in CRT Raster Units

\begin{tabular}{ccc}
\hline & Condition 1 & Condition 2 \\
\hline-21 & 70 \\
-83 & 19 \\
-15 & -16 \\
32 & 98 \\
39 & 2 \\
& -16 & 73 \\
& -67 & 226 \\
& 13 & 20 \\
& 15 & 145 \\
& -23 & -102 \\
7 & 95 \\
& 28 & 163 \\
& 76 & 110 \\
Mean & 48 & 71 \\
SD & -19 & 81 \\
\hline
\end{tabular}

Note-35 raster units $=1^{\circ}$.

Condition 1 group was not significantly different from zero $[t(14)=.09]$. For Condition 2 , the difference was significant $[\mathrm{t}(14)=3.43, \mathrm{p}<.01]$.

\section{Discussion}

The results clearly favor potentiation theory, but it had not been expected that no evidence of any adaptation under Condition 1 would be found. A previous experiment by the authors (Gourlay, Gyr, Walters, \& Willey, 1975) had shown adaptation to curvature using a similar CRT-simulation methodology and under conditions in which it is unlikely that potentiation could have played a part. Two possibilities for the failure were considered. First, it was thought than an adaptation score based on a fairly large number of measures ( 24 pretests and 24 posttests) might have been affected by decay effects. However, a test based on adaptation means computed from only the first four posttest scores, rather than the entire 24, revealed no support for this possibility [mean $=-12.87, \mathrm{SD}=57.55, \mathrm{t}(14)=-0.87$ ] Secondly, it was felt that the cue discrepancy might not have been direct or compelling enough, since the subject did not actually see his hand, but only a simulation of its (supposed) location. To meet this objection, a second version of the experiment was run, eliminating the simulation, using actual prisms, and allowing the subject the sight of his fingers during training.

\section{EXPERIMENT 2}

\section{Method}

Subjects. As in Experiment 1,15 subjects, each required to have normal vision with or without glasses, were used in each condition.

Apparatus. The apparatus was basically the same as in Experiment 1 , except for the following modifications: (1) The hood was replaced by a stereoscope hood in which prisms could be mounted. Two 12-diopter prisms were used, which gave nearly the same displacement as in the simulation. (2) The horizontal panel was moved back slightly, so that the subject could see his fingers just as they reached the screen. A light was placed above the CRT, allowing a clear view of the fingers. (3) To prevent sight of the CRT boundaries while the light was on during training (which would have revealed that the stimuli were asymetrically placed with respect to these boundaries), a tube, $20 \mathrm{~cm}$ in length and $10 \mathrm{~cm}$ in diameter, was positioned in front of the stereoscope hood. The angle of this tube could be adjusted so that the stimuli were centered within it. (4) The simulated prism displacement was removed from the program (this affected only Condition 1; the displacement designed to induce potentiation without prisms was retained for Condition 2).

Procedure. The pretest and posttest procedure was the same as in Experiment 1, except the prisms were in place for both tests in Condition 1 and were removed for Condition 2. With the light out and the filter in place, there were no extraneous features, such as color fringes, which differentiated the conditions.

At the end of the pretest, the subject left the booth temporarily, and the experimenter then turned the light on, removed the filter, and positioned the tube in front of the hood (the tube had not been in position during the pretest, as, in darkness, it was not needed). The tube was centered on the first training stimulus, which, for Condition 1, was equal to the mean of the subject's pretest (but, as in the pretest, seen through prisms), and for Condition 2, was equal to the pretest mean plus the rightward displacement of $6.69^{\circ}$ (but, as in the pretest, not seen through prisms). Training proceeded as in Experiment 1, except that the subject could now see his fingers holding the light pen just as his hands reached the screen (this feedback occurred only at the end of the hand movement, which, as before, occurred without visual guidance). At the end of training, the light was turned off, the tube removed, and the filter repositioned in preparation for the posttest. The apparatus was arranged so that these steps could be performed very quickly, thus avoiding decay problems.

\section{Results}

Computation of adaptation scores was performed as in Experiment 1. The results are shown in Table 2. The pattern is clearly similar to that in Experiment 1,

Table 2

Experiment 2 Adaptation Scores in CRT Raster Units

\begin{tabular}{lcc}
\hline & Condition 1 & Condition 2 \\
\hline 201 & 9 \\
21 & 49 \\
-9 & 157 \\
-90 & 102 \\
26 & 64 \\
47 & 97 \\
-1 & -11 \\
& -76 & -3 \\
& 4 & 152 \\
& 2 & 29 \\
& 89 & 92 \\
& -24 & -76 \\
& 40 & 168 \\
Mean & -78 & 14 \\
SD & -6 & 153 \\
\hline
\end{tabular}

Note-35 raster units $=1^{\circ}$. 
and there is no evidence that the change in procedure induced any adaptation in Condition 1. The mean of Condition 1, although positive, is not significantly different from zero $[t(14)=.52]$. The mean of Condition 2 is significantly different from zero $[t(14)$ $=3.51, \mathrm{p}<.01]$. The difference between the groups is significant $[\mathrm{t}(28)=2.14, \mathrm{p}<.05]$. As before, adaptation scores for Condition 1, based on the first four posttest scores, yielded no evidence for adaptation which might have been present early in the posttest but subject to decay $[\mathrm{m}=-8.13, \mathrm{SD}=72.03$, $\mathrm{t}(14)=-0.44]$.

\section{Discussion}

The results of Experiment 2 provide additional evidence of potentiation effects, and further support the suggestion made by Ebenholtz that potentiation may account entirely for prism adaptation (Ebenholtz, 1974; Ebenholtz \& Wolfson, 1975; Paap \& Ebenholtz, 1976). However, as noted previously, this latter contention seems at odds with a previous experiment by the authors (Gourlay, Gyr, Walters, \& Willey, 1975). It has also been challenged recently by Craske and Crawshaw (1975) and by Wallach and Halperin (1977). These criticisms will be reviewed briefly.

Craske and Crawshaw set up an exposure condition in which prism displacement was to the left, whereas the eyes were required to deviate to the right, thus leading to opposite predictions of the direction of the effect for potentiation and recalibration theory. During exposure, the subjects, while looking at their feet, had their heads held $25^{\circ}$ to the left by means of a biteboard. The leftward prism displacement was less than this, so the eyes had to deviate about $13.7^{\circ}$ to the right in order to see the feet. Results uniformly supported the recalibration prediction. However, this conclusion depends on the assumption that potentiation of the neck muscles was not involved. The net displacement of the head-eye system is to the left, so that, if the entire system is involved in the judgment of direction, potentiation and recalibration theory are once again confounded. Craske and Crawshaw report a reference claiming to have found no evidence for head adaptation (Crawshaw \& Craske, 1976). In their Experiment II, subjects looked at their feet through prisms and then judged the position of their noses; no shift in judgments was found. However, in this experiment, the head appears not to have been constrained by a biteboard during exposure (it was constrained only during testing in Experiment I, and no difference in this respect is reported among the specifically listed changes made for Experiment II). Under these conditions, the subjects may have deviated only their eyes during exposure, and all the potentiation may have been in the eye muscle system, for which there was no test. Thus, the data of Craske and Crawshaw do not con- vincingly challenge the claims of potentiation theory.

Wallach and Halperin (1977) offer stronger evidence for effects not accountable by potentiation theory, though they do not deny that potentiation exists and, in fact, note that one of their experiments $(1 \mathrm{~b}$, in which only potentiation effects could have occurred) supports Ebenholtz and Wolfson (1975, Experiment 2). However, Wallach and Halperin are dealing, not with adaptation to displacement, but with adaptive shifts in size and distance perception resulting from altered convergence.

What seems to be a reasonable set of conclusions is that (1) potentiation certainly exists and may have been confounded with other effects in the experimental literature, but that (2) recalibration probably exists in some cases, but is a more complex and varied phenomenon, depending strongly for its strength or existence on the nature and perceptual significance of the cue conflicts involved. Thus, in the present experiments, cue conflict was between the felt position of (or efferent directions to) the arm and the visual perception (or representation) of the fingers; in the Wallach and Halperin experiments, cue conflict was between altered convergence and other cues to size and distance. It is reasonable, a priori, that the latter situation might produce a stronger demand on the perceptual system for cue conflict resolution. We do not perceive the same object at two different distances, or as having two different sizes, at the same time, but it is possible (though the experience would be regarded as odd and illusory) for one to feel as if one's arm is at a different place from where it is seen, and it is certainly possible for one to think one has aimed at one place and find that one has hit another. In the present experiments, 8 Condition 1 subjects in Experiment 1 and 10 Condition 1 subjects in Experiment 2 reported, in postexperimental interviews, some sense of vision-arm discrepancy during training, though their remarks were often difficult to interpret. Perhaps this discrepancy is simply not paradoxical enough for the perceptual system to demand resolution, so that, in this experimental situation, adaptation effects are solely due to potentiation. This may not be the case in other situations.

\section{REFERENCES}

Craske, B., \& Crawshaw, M. Oculomotor adaptation to prisms is not simply a muscle potentiation effect. Perception \& Psychophysics, 1975, 18, 105-106.

Crawshaw, M., \& CRASKE, B. Oculomotor adaptation to prisms: Complete transfer between the eyes. British Journal of Psychology, 1976, 67, 475-478.

Ebrnholtz, S. M. The possible role of eye-muscle potentiation in several forms of prism adaptation. Perception, 1974, 3 , 477-485.

Ebenholtz, S. M., \& Wolfson, D. M. Perceptual after effects of sustained convergence. Perception \& Psychophysics, 1975, 17, 485-491. 
EPSTEIN, W. Recalibration by pairing: A process of perceptual learning. Perception, 1975, 4, 59-72.

Gourlay, K., GYr, J. W., Walters, S., \& Willey, R. Instrumentation designed to simulate the effects of prisms used in studies of visual rearrangement. Behavior Research Methods \& Instrumentation, 1975, 7, 294-300.

PaAp, K. R., \& Ebenhol.Tz, S. M. Perceptual consequences of potentiation in the extraocular muscles: An alternative explanation for adaptation to wedge prisms. Journal of Experimental Psychology: Human Perception and Performance, 1976, 2, 457-468.
Rock, I. The nature of perceptual adaptation. New York: Basic Books, 1966.

Wallach, H., \& Frey, K. J. On counteradaptation. Perception \& Psychophysics, 1972, 11, 161-165.

Wallach, H., \& Halperin, P. Eye muscle potentiation does not account for adaptation in distance perception based on oculomotor cues. Perception \& Psychophysics, 1977, 22, 427-430.

(Received for publication December 6, 1977; accepted revision received June 22,1978 .) 